\title{
Midwives' Perceptions of Barriers to Respectful Maternity Care for Adolescent Mothers in Jamaica: A Qualitative Study
}

\author{
Karline Wilson-Mitchell, Amy Marowitz, and Jody R. Lori
}

BACKGROUND: Few studies have examined respectful maternity care (RMC) of adolescent mothers in the Caribbean. The researchers' objective was to explore the perceptions of midwives who provide care for adolescent mothers in Jamaica. Design: A pilot qualitative study was conducted in 2015. Participants were 12 community or nurse-midwives who practiced in rural and urban regions of Jamaica attended a nursing and midwifery conference.

METHOD: Semistructured focus groups and interviews of Jamaican midwives were conducted. The World Health Organization (WHO) definitions of respectful care in relationship to sexual and reproductive health rights, and data were examined using a poststructural feminist version of Denzin's interpretive interactionism theory. Braun and Clark's method of reiterative content analysis was employed.

FINDINGS: Participants shared their work experience with adolescent mothers and explained that restrictive public and institutional policies culture, personal beliefs, and the location of care delivery hindered them from providing respectful care which allows for shared decision-making, informed consent, and allowing for a desired labour companion. They perceived their roles as advocates, educators, expert helpers, and worker activists.

CONCLUSIONS: Out of hospital environments appeared to facilitate the advocacy role. Policies and attitudes had the potential to hinder or to promote RMC. These findings may be used to inform a national midwifery survey, educational modules, health systems change to allow for less restrictive policies and spaces.

KEYWORDS: developing countries; midwives; adolescent; maternity care; rights; quality care

\section{INTRODUCTION}

Respectful maternity care (RMC) has been prioritized by nursing associations and regulators, global health researchers, policy makers, and women's advocacy groups (Dao, 2012; Gee \& Corry, 2012; Miller et al., 2016; Alliance, 2015). Adolescent mothers in the Global South have described disrespectful care (Abuya et al., 20152012; Bowser \& Hill, 2010; Wilson-Mitchell, Bennett, \& Stennett, 2014). Few studies have examined RMC delivered to adolescent mothers in the Caribbean.
Still fewer describe the opinions and perspectives of Jamaican midwives and perinatal nurses concerning this issue.

\section{REVIEW OF THE LITERATURE}

\section{Measuring Respectful Maternity Care}

Prior to 2015, the concept of respectful care was difficult to measure. It was often implied when researchers 
examined related issues of patient satisfaction, highquality humanistic care, and freedom from abuse (Bowser \& Hill, 2010). Respect appeared to be a subjective and personal goal hoped for, noticeable when it was absent, an ethical gold standard that healthcare professionals aspired to, but difficult to measure when it was achieved. Fortunately the World Health Organization (WHO) developed broad definitions of respectful care that provide objective, concrete parameters by which researchers, policy makers, and educators can observe and measure globally what might otherwise appear to be nebulous and subjective outcomes of quality maternity care (WHO, 2015b). The seven domains for measuring respectful care, comprising various components, were identified by WHO and include: (a) freedom from physical harm and ill treatment; (b) informed consent and refusal and shared decision-making, including choice of a support person in labor; (c) privacy and confidentiality; (d) dignity and respect, including freedom to change positions for labour or birth; (e) equality, freedom from discrimination, and equitable care; (f) access to the highest quality health care available; (g) liberty, autonomy, self-determination, and freedom from detention or coercion. From these domains, the focus of this study was to examine components of domains b, c, d, and f, namely informed consent, shared decision-making, confidentiality, and the right to have a companion in labor (Bowser \& Hill, 2010; WHO, 2015a).

Using these four components, the literature is replete with examples of disrespectful care by maternal care providers in low-resource countries such as the Dominican Republic (Miller et al., 2003), India (Bowser \& Hill, 2010; Singh, Rai, Alagarajan, \& Singh, 2012), Kenya (Abuya et al., 2015; Bohren et al., 2015), Peru, Burundi, South Africa (Bowser \& Hill, 2010), Nigeria, and Tanzania (Bohren et al., 2015). Many of these studies included mothers ages 15-45 with an unspecified proportion of adolescent mothers (Bowser \& Hill, 2010). Despite the existence of human rights laws in many countries, the types of behaviors observed include: breaking confidentiality about HIV status; shouting or screaming for example when the client makes a request or attempts to change positions; name-calling with clients of a different ethnicity or tribe; abandonment when the client declines a cervical exam (leaving her to birth unattended); episiotomy and cesarean section without consent; barring the client's partner or mother from providing labor support when the space would safely permit accompaniment (Bowser \& Hill, 2010; Miller et al., 2016). In randomized control studies (RCTs) with clustered sampling of 506 villages, interventions such as deploying community workers who are skilled in cultural sensitivity and respectful communication strategies improved maternal satisfaction, service utilization, and perinatal outcomes in many cases (Bhutta et al., 2011). Prost et al. (2013) published a systematic review of seven clustered RCTs with samples ranging from 3,303 to 15,257 ; they found a statistically significant reduction in perinatal mortality in five out of the seven studies when respectful care, interactive and structured group prenatal care were employed. Participatory group interventions are complex and it is difficult to determine which component of the intervention had the greatest effect. Notably, some studies also identified disrespectful care in the Global North, including Australia and Canada (Bowser \& Hill, 2010; Small, Yelland, Lumley, Brown, \& Liamputtong, 2002).

The mixed-method desktop review by Bowser and Hill (2010) surveyed gray literature as well as quantitative and qualitative studies to identify findings related to disrespect, abuse, humanized birth, childbirth rights, treatment in emergencies, health systems in childbirth settings, structural barriers or discrimination, health workers, and empowerment during childbirth from both the mothers' and the workers' points of view. A wide cross section of multidisciplinary works was reviewed. Documents from 18 nations were reviewed including: Canada, United States, Burkina Faso, Ghana, Sierra Leone, Kenya, Burundi, Tanzania, Zimbabwe, Sudan, India, Pakistan, Brazil, Peru, Dominican Republic, Brazil, and Lebanon. The review also included interviews from key experts in maternal health policy. The review of Bowser et al. is one of the first to highlight adolescents as a vulnerable population constituting a subset of the mothers who experience disrespectful care and abuse. This report is seminal for its breadth, the quality of the studies and interviews synthesized, and the policy implications that were concluded and implemented at its completion.

A recent study by Thompson, Land, CamachoHubner, and Fullerton (2015) performed a rapid assessment of 83 maternity health workers in eight Latin American and Caribbean countries (including Guyana) which included 23 midwives using the WHO childbirth rights and respectful care framework. Mixed methods were used, including expert observations of clinical care throughout the perinatal period using reliable study instruments, chart reviews, 
interviews of staff and patients after discharge, and perinatal outcome records. Although midwives in the study tended to have higher quality care and competency ratings, they remained below expected WHO and International Confederation of Midwives (ICM) goals for promoting privacy, encouraging a supportive companion, providing information on progress of labor, and supporting maternal-newborn bonding and breastfeeding.

\section{Experiences of Midwives in the Developing World}

Midwives in low-resource countries report challenging shortages of equipment and staff, job dissatisfaction, low morale or motivation, significant desire to quit, and inadequate training (Abuya et al., 20152012; Bohren et al., 2015; Bonenberger, Aikins, Akweongo, \& Wyss, 2014; Knight, Self, \& Kennedy, 2013; Miller et al., 2003). Researchers concluded that these factors were associated with higher maternal and neonatal mortality rates. Abuya et al., 20152012 conducted a mixed-methods study of 13 Kenyan facilities, sampling 641 mothers who received care from a multidisciplinary team that included midwives. The researchers noted a significantly greater amount of disrespect and abuse from providers was observed in multiparous, married, lower socioeconomic mothers and ranged from facial expressions to demands of bribes or physical abuse. The mothers identified their perceptions of satisfying birth as birth that was not humiliating (Abuya et al., 20152012).

Bonenberger et al. (2014) conducted a cross-sectional survey study of Ghanian 256 health-care workers (13.3\% of which were nurse-midwives) in which the dependent variables were job satisfaction, motivation, desire to quit, burnout, and organizational commitment using a 5-point Likert-like scale. Although the modified scales were not validated, logistic regression revealed that midwives were more likely to have decreased motivation and job satisfaction (adjusted odds ration of 3.16) compared to physicians (adjusted odds ratio 1.32) or registered nurses (adjusted odds ratio 1.0). The mixed-methods systematic review of 43 African, Asian, and Latin American studies by Knight et al. (2013) noted health-care delays, barriers to accessing care, and marked job dissatisfaction among midwives. In 33 of these studies, there was a negative correlation found between adverse outcomes such as perinatal mortality and adequate training, drug supplies, working equipment, and staff motivation. The researchers found that $65 \%$ of the time there were logistics or supply problems;
$60 \%$ of the time there were staff shortages; $51 \%$ of the time there was lack of equipment of non-functioning equipment; and $44 \%$ of the time there were problems with staff morale. Unfortunately, the details which could distinguish low from middle-income nations was lacking; however, as many as 626 health workers across the disciplines of nursing, midwifery, pharmacy, and allied staff were interviewed. The findings from these studies are compelling in terms of the relationship between staff morale, poor working conditions, and subsequent poor perinatal outcomes.

\section{Experiences of Mothers in the Developing World}

Adolescents were among those who describe disrespectful care that deterred them from receiving antenatal or intrapartum care in many of the qualitative studies (Atuyambe, Mirembe, Annika, Kirumira, \& Faxelid, 2009; James, Rall, \& Strümpher, 2012; Oyerinde et al., 2013; Shiferaw, Spigt, Godefrooij, Melkamu, \& Tekie, 2013; Small et al., 2002 and Wilson-Mitchell et al., 2014). These studies were conducted in several African countries, Latin America, Jordan, Canada, Australia, the Dominican Republic, Sierra Leone, and one from Jamaica. Mothers reported the following experiences in these studies: mothers avoided care due to disrespectful behaviors of the caregivers; they were denied adequate informed consent when they desired it; they were not included in the decision-making process surrounding admission and plan of care and had little understanding of rationale for interventions; they experienced violations of privacy, personal information or modesty; they expressed a desire to have a known labor support companion but restrictive institutional policies were described in many cases.

The mixed-methods study by (Miller et al., 2003) in the Dominican included the focus groups and interviews of 57 prenatal patients, 55 women in labor, 21 women with vaginal births, and six women following cesarean sections. They described a noisy, foul-smelling environment characterized by a lack of help from nursing, being rushed through procedures, unnecessary and forced nudity, students talking around them as if they were invisible, being blamed for their baby's complications and death, and a lack of informed consent prior to cesarean sections (Miller et al., 2003, p. 99).

An Ethiopian mixed-methods study speaks to the importance of acceptable care that is centered around the desires and worldview of the woman (Shiferaw et al., 2013). The study employed surveys, focus groups, 
and individual interviews of 909 women in various Ethiopian villages where trained health-care providers (nurses, midwives, and physicians) were available. Although most of the women accessed adequate antenatal care (at least three visits) from these providers, 78\% of them preferred to have homebirth with traditional birth attendants. In-depth interviews revealed that they believed the trained health-care providers were insensitive to their customary practices, did not support family members during delivery, were incompetent, were unavailable, were not respectful of privacy and did not provide psychosocial support.

Duggan and Adejumo (2011) conducted a qualitative study of 18 adolescents from three racial groups in South Africa. The participants explained that respect involved encouragement, pleasant greetings, accommodating of their discomforts, and taking their reports seriously when they sensed that the second stage of labor had begun. They reported that a feeling of safety to discuss their private information with assurance of confidentiality, gentle, engaging body language, and health information crucial to preparation for parenting were all desirable aspects of care they expected. Participants were discouraged by the lengthy clinic wait times. Researchers concluded that providers were important conduits that helped adolescent mothers to navigate the health-care system and recommended further study to determine how ethno-racial factors might influence service utilization (Duggan \& Adejumo, 2012).

James et al. (2012) conducted a qualitative study of 12 adolescent mothers' experiences of antenatal care in South Africa. The main theme discovered was that "the ante-natal care clinic environment was alienating to them" (James et al., 2012). They were embarrassed, judged, and humiliated by the perceived superiority displayed by the adult patients and their discomforts posed a barrier to coming to clinic. The study was limited by the lack of standardized reporting of demographics (Wilson-Mitchell \& Wilson-Mitchell, 20152015).

One of the few Caribbean studies of adolescent mothers' perceptions was conducted by (WilsonMitchell et al., 2014; Wilson-Mitchell, Stevens, Wilson-Mitchell, \& Stevens, 20142014). In this qualitative study, 45 adolescent mothers' experiences with standard care among adult mothers were compared to experiences attending a pilot teen clinic with a separate waiting room for teens, where social and mental health referrals were readily available. Some of the mothers were recipients of harsh instructions, public humiliation from staff, and verbal abuse from adult patients, while others reported being treated politely and warmly by staff (Wilson-Mitchell et al., 2014; Wilson-Mitchell et al., 20142014). Recommendations from this study included the adoption of separate adolescent-centered care delivery models for all facilities, structured group prenatal care, and policy changes to allow adolescent mothers to be accompanied by a labor support companion (Wilson-Mitchell et al., 20142014).

\section{Cultural Context}

The Jamaican adolescent pregnancy rate is 72 per thousand comprising $18 \%$ of all Jamaican pregnancies (United Nations Population Fund, 2013). These mothers are stigmatized and experience conflicted relationships with their own mothers (Hutchinson et al., 2012; Wilson-Mitchell et al., 2014). Health care is delivered in a two-tiered system (private and publicly funded). Adolescent pregnancy is considered a high risk condition and minors are only emancipated to self-consent after the age of 16 .

Adolescents often avoid seeking prenatal care early due to the fears of discovery and expulsion from their neighborhood school (Wilson-Mitchell et al., 20142014). Early engagement decreases risks of untreated complications, for example, preterm labor or gestational hypertension (Vieira et al., 2012). It has been estimated by regional policy analysts that Jamaica loses 21,582 disability-adjusted-life-years (DALYs) per 100,000 births and 273,711 DALYs per 100,000 neonates managed without EBM (Murray et al., 2012). A DALY is the sum of life lost and years lived with disability for 291 causes. Long-term adolescent births cost taxpayers $\$ 2,000$ USD per year and $12.3 \%$ of the GDP according to leaders in the Caribbean and Latin American regions (Aguilar, 2015). Such a cost-benefit analysis lends support to making quality improvements to the current system of adolescent care.

\section{Purpose}

The purpose of this study was to explore the opinions and perspectives of Jamaican midwives and perinatal nurses regarding barriers to provision of respectful, quality maternity care of Jamaican adolescents.

\section{METHODOLOGY}

Ethics approval was obtained from Ryerson and Frontier Nursing Universities. Purposive sampling was used to recruit participants who were midwives or 
nurse-midwives from all Jamaican parishes who met the inclusion criteria and attended the Caribbean School of Nursing, University of Technology, Jamaica 2nd Biennial Nursing and Midwifery Research Conference, August 13-14, at the Holiday Inn Sunspree Resort in Montego Bay, Jamaica. In addition, advertising flyers were emailed to the President of the Nurses Association of Jamaica, the President of the Jamaica Midwives Association, the Director of the Midwifery Program at $\mathrm{U}$ Tech, and distributed at the conference. Participants completed informed written consents prior to interviews. Attending the conference as part of the inclusion criteria enabled the researcher to use the conference venue and the preexisting communication network for focus groups and interviews.

The theoretical framework used to develop data collection and analyze findings from this study was a critical poststructural feminist version of Denzin's theory of interpretive interactionism (Denzin, 2001; Jefford \& Sundin, 2013; Sundin \& Fahy, 2008). This framework was ideal because it clearly identified "critical turning points" in the challenging and austere work conditions and the interactive processes of the midwives' work experience in the developing world. The feminist lens allows researchers to ask questions about childbirth rights and workers' rights which explore power imbalances during these significant events of the midwife's work (Jefford \& Sundin, 2013). Figure 1 provides a representation of the significant symbolic interactions in the Jamaican midwife's experience of work. A feminist model explores power relationships between all members of a system or situation. Consequently, the powers held by employers (i.e., the Ministry of Health $[\mathrm{MoH}]$ ) over workers, policy makers over midwives, midwives over clients are explored using the model. There are pivotal work experiences that midwives encounter because of the power relationships in which they live and work. This model utilizes a more ecological approach to look at all symbolic relationships simultaneously, thus considering the potential conflicts, stressors, obligations, or responsibilities that the midwife must balance in a dynamic environment on a daily basis.

Description of the ample is displayed in Table 1 with demographics for the 12 midwives from two focus groups with respect to age group, type of education, current practice, years of experience, and average number of adolescent clients cared for weekly. The average age of the midwives was 41.5 years and the average duration of practice in nursing and/or midwifery was 12 years, although the rural midwives tended to have a longer work experience. The midwives came from six of Jamaica's 14 parishes (geographical regions) with equal numbers coming from rural and urban practices. All were female employees of the MoH, Jamaica. The urban midwives were primarily baccalaureate-prepared nursemidwives who worked in low-risk, high-risk acute care, antepartum, intrapartum, postpartum, community health, and family planning. Some also supervised

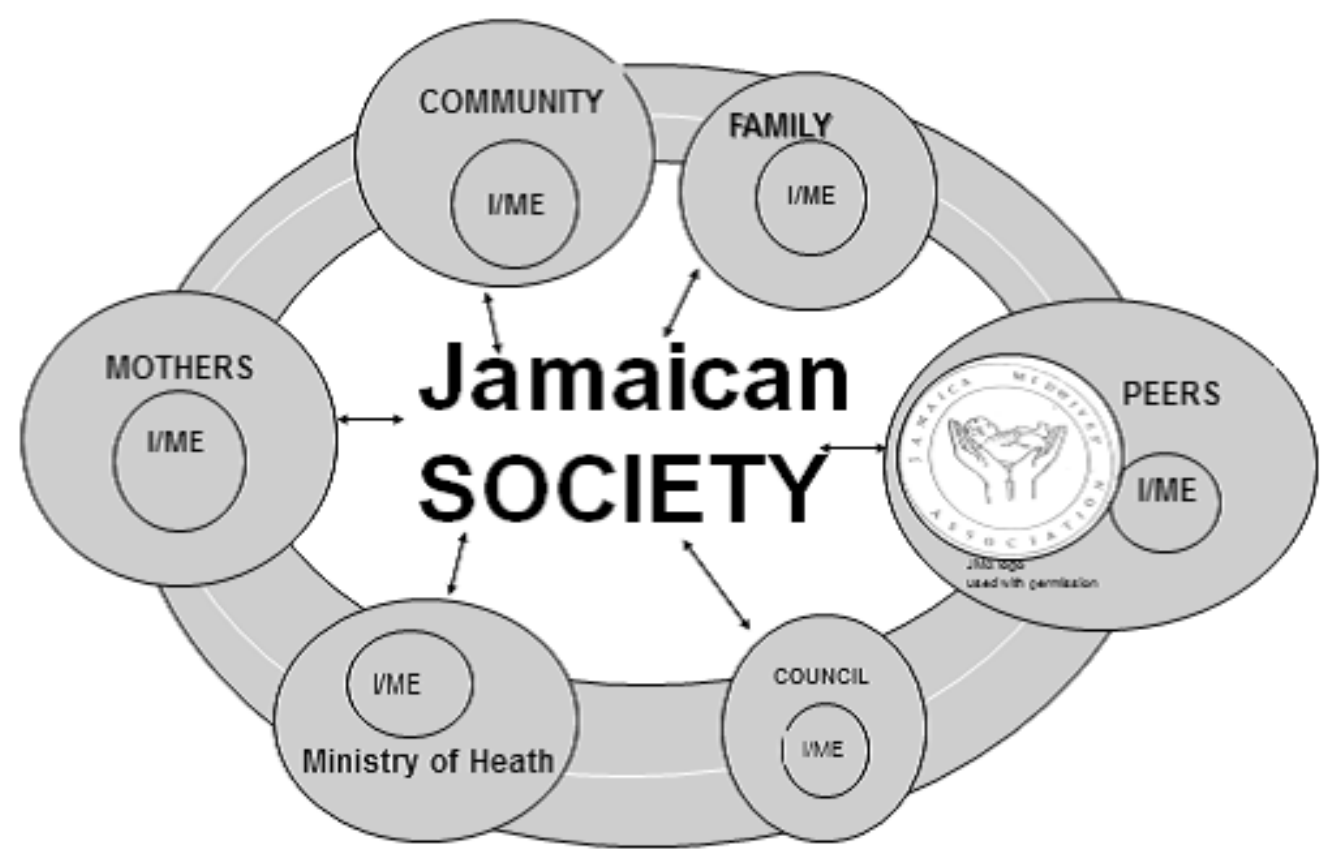

FIGURE 1. Significant symbolic interactions of Jamaican midwives. Source: Wilson-Mitchell (2016). Note: Ministry of Health (MoH), Jamaica Midwives Association (JMA), JMA logo used with permission. 
TABLE 1. DESCRIPTION OF SAMPLE

\begin{tabular}{|llll} 
& URBAN & RURAL & \\
& MIDWIVES & MIDWIVES & TOTAL \\
MEMOGRAPHIC & MEANS (RANGE) & $\begin{array}{l}\text { MEANS } \\
\text { (RANGE) }\end{array}$ & MEANS (RANGE) \\
\hline Age (years) & $38.2(32-42)$ & $37.5(34-58)$ & $41.5(34-58)$ \\
Duration of practice (years) & $8.2(5-15)$ & $15.8(1-25)$ & $12(1-36)$ \\
Professional education (years) & $5(4-5)$ & 2 & $3.25(2-5)$ \\
Number of adolescent mothers & $1-5$ & $1-5$ & $2.5(1-5)$ \\
\hline \multicolumn{1}{c}{ assigned per week } & & & \\
\hline
\end{tabular}

students or worked as contract instructors for University of Technology (UTech). The majority of the midwives (seven) were rural midwives who were certificate-prepared community midwives. They primarily conducted low-risk home births, well-woman, and primary care in community health centers. The rural midwives were eager to enroll in the UTech bridging program that would provide a midwifery baccalaureate with increased prestige and potentially increased remuneration.

The five 4-year baccalaureate prepared midwives completed their nursing education followed by a 1-year specialization in midwifery that was attached to a teaching hospital (University of West Indies). Most of the nurse-midwives practiced to the fullest scope in Jamaica, which involved maternity, reproductive health, primary, and newborn care. Two of the midwives had previously managed antepartum and intrapartum care; however at the time of data collection, they worked in an intensive care unit in an obstetric nurse role in which high-risk antepartum and postpartum clients received care. One midwife worked in a neonatal intensive care unit. Two midwives managed antenatal care in a hospital-based clinic which included high-risk patient management (including well-adolescents who were categorized as high-risk patients in Jamaica). Seven midwives who practiced in rural community primary care clinics provided primary care as well as midwifery care but focused on maternity, newborn care, and infant care up to the age of 2 years.

Two semistructured focus group interviews in a hotel meeting room and two subsequent interviews of focus group participants, lasting between 45 and 60 minutes, were completed to discuss the following five research questions regarding: (a) perceptions of role as midwife, (b) opinions and thoughts about informed consent regardless of age, (c) thoughts about shared decision-making with a teen mother, (d) houghts surrounding a support person accompanying the teen mother, and (e) thoughts surrounding privacy. These questions were accompanied by definitions based upon the WHO definitions of respectful care and the research/ discussion facilitator adhered to current focus group recommendations (Morgan, 1998) and use of examples to elaborate on meanings whenever requested.

The digital audio recordings of the focus groups and individual interviews were clear and audible for the most part. Field notes were very helpful and compensated for the inability to video-record. NVIVO files (consent, recordings, questionnaires, field notes) were encrypted and password-protected in a locked office. There were no breaches of privacy or security. The analysis of the data was facilitated using NVIVO (10th version) coding. Fortunately, the midwives were proficient in a level of English so that NVIVO coding of phrases and meanings were straightforward (although the Patois dialect and English are spoken interchangeably by this population).

The following strategies were used to optimize rigor or trustworthiness in the analysis (Shenton, 2004; Tuckett, 2005). To optimize dependability and confirmability, records were kept of the recorded statements of each participant and the researcher ensured that each transcription could be connected to its original recordings in NVIVO 10. For this pilot, the research also played the role of the transcriber. The process for the identification of themes, coding, and axial identification was recorded in the NVIVO searches and memos as well as field notes. Dependability is improved by noting the date, time and thinking behind decisions made. Notations were made in memos, field notes, recordings and thematic logs which are stored in NVIVO.

The Braun and Clarke (2006) reiterative method of thematic analysis was used. It involved an initial reading of the transcripts and field notes to allow immersion once again in the experience to recall facial expressions and meanings of tone and pauses. Then a second reading helped to initiate coding. Subsequent readings allowed connection of codes to begin to develop themes. Finally, what emerged was a conceptual diagram that helped to articulate the key players 
TABLE 2. Thematic Analysis

\begin{tabular}{|c|c|c|}
\hline THEME & EXPRESSIONS & $\begin{array}{l}\text { QUALITY IMPROVEMENT } \\
\text { IMPLICATIONS }\end{array}$ \\
\hline Perceived roles of the midwife & $\begin{array}{l}\text { Advocate } \\
\text { Educator } \\
\text { Expert helper } \\
\text { Activist-worker }\end{array}$ & $\begin{array}{l}\text { Capacity building, continuing educa- } \\
\text { tion re. respectful care (Jhpiego, } \\
\text { White Ribbon Alliance curricula) } \\
\text { Conflict resolution skills building }\end{array}$ \\
\hline The impact of culture & $\begin{array}{l}\text { Patriarchalism } \\
\text { Parental authority } \\
\text { Adolescent as vulnerable child } \\
\text { Sexuality \& reproductive = decisions } \\
\quad \text { adult domain } \\
\text { Perceived asexuality of adolescence } \\
\text { Judeo-Christian ethic \& belief } \\
\text { System } \\
\text { Freedom, autonomy, and self-determina- } \\
\text { tion based upon age } \\
\text { Rights and privilege that are not } \\
\text { universal }\end{array}$ & $\begin{array}{l}\text { Continuing education support, building } \\
\text { capacity, and strengthening profes- } \\
\text { sion through Association of Jamaican } \\
\text { Midwives and Association of Jamaican } \\
\text { Nurses according to International } \\
\text { Confederation of Midwives priorities. } \\
\text { Engage community-based participa- } \\
\text { tory research partners in developing } \\
\text { respectful maternity care evidence that } \\
\text { is specific to Jamaican context. }\end{array}$ \\
\hline The impact of policy and place & $\begin{array}{l}\text { Paternalism and hierarchy in governmen- } \\
\text { tal agencies } \\
\text { Employee model of midwifery } \\
\text { Policy adherence dependent upon privi- } \\
\text { lege and status } \\
\text { Bending the rules outside of the } \\
\text { hospital } \\
\text { Policy confines and restricts both midwife } \\
\text { and mother } \\
\text { Bending the rules out of hospital } \\
\text { Different standards and expectations } \\
\text { determined by place of work or place } \\
\text { of birth }\end{array}$ & $\begin{array}{l}\text { Plan Do Study Act (PDSA) cycle for } \\
\text { policy change within the Ministry } \\
\text { of Health and Ministry of Youth and } \\
\text { Culture to create public and health } \\
\text { policies informed by social justice, } \\
\text { health equity and respectful maternity } \\
\text { care principles. } \\
\text { Community partnership building through } \\
\text { academic and non-governmental } \\
\text { organizations. }\end{array}$ \\
\hline
\end{tabular}

or elements and themes discovered in the preliminary analysis.

\section{Findings}

The data were rich with personal stories, information about health policy, situations of dilemma and confusion as well as detailed descriptions of the midwifery role as they perceived it. The following themes emerged from the preliminary analysis with representative overarching themes dealing with the midwife or adolescent's position in the system (Table 2): (a) perceived roles of the midwife; (b) the impact of culture; (c) the impact of policy and place. These themes were examined in terms of their influence as a facilitator or a barrier to respectful care in terms of informed consent, shared decision-making, provision of privacy and/or confidentiality, and allowing a desired labor support companion. In addition, Figure 2 is a depiction of the maternity care system or a graphic model of how the midwives saw themselves and the mothers positioned both in terms of place and policy. Both the midwife and the client were constrained by the same public and facility policies; consequently both were subject to the power of policy and place. Location in the facility/hospital determines how much freedom, agency, and creativity can be employed to deliver RMC. Indeed, these issues of control, power, and agency in the health-care facility were more easily analyzed through a feminist lens, particularly since both the clients and midwives were primarily female.

\section{Perceived Roles of the Midwife}

Within the theme "perceived roles of the midwife," the participants described the roles of advocate, educator, expert helper, and activist-worker. They spoke proudly of these roles, and most often the roles facilitated the provision of information required for consent, prevented them from abandoning a mother who desired an out-of-hospital birth, or support for 


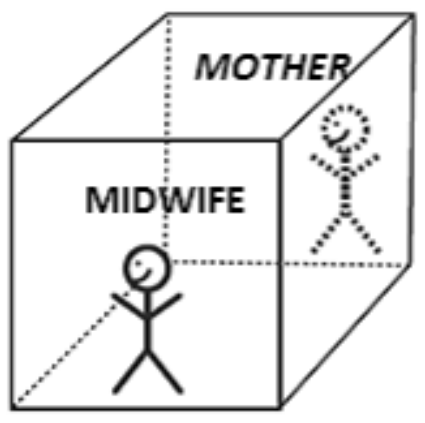

\section{PLACE \\ Home/hospital}

FIGURE 2. Mother's and midwife's position in the health-care system. Note. Modified with permission from image titled: "Person In Cube" at https://www.learner.org/courses/mathilluminated/units/4/textbook/04.php.

a desired labor companion when hospital policies allowed. Following are descriptions of the advocate role.

...we're the only one who will be able to reach the unreachable. And the laws are only there on paper... [What we can do] to assist these individuals! (P3)

Yes, the mother was her center. Yes, the mother (of the patient) being there, you would not believe and we've proved that over and over. Support during labor, it helps. We've seen that. Proven that in our home deliveries. (P1)

The role of advocacy seemed to engender the adolescents' trust. It also promoted the provision of privacy and protection of confidentiality.

It's the relationship that you have with them. When they come to the health center they are looking for you to be a confidential person because most of the time they confide in you... [I] t's like you're trying to make the health center a home for them. So... we tell them that we are all one family. Whatever is said here, will stay here... [T] hey get to develop a sense of relation with you where they are comfortable, $[\ldots]$ they may on a whole put their trust in you [...] most of the time you tend to be sorry for them. Because most of them, the background that they have, family background is not really good. (P4)
The midwives repeatedly shared that the educator role was an important role in which support and advocacy were promoted by information sharing.

[Sometimes]... they are timid... shy... uncomfortable because already they're expecting you to be cussing them and treating them bad, as I guess the parents do. So you have to comfort them first as a sort of a counselor. Then you have to explain to them... actually teaching them about the development of their body... changes... during the labor and the delivery. So you are actually teaching them. Sometimes you will have to be a friend, because they can't relate to their parents or their peer groups any more. (P2)

$[\ldots]$ they are more receptive to information. And if they come in with preconceived ideas, especially in labor, if you have something set and you discuss it, and if there's a need for a change and something arises that you have to change the plan ... (P5)

Many midwives expressed pride in taking the role of an expert helper, whose clinical management skills were highly prized. Care was provided in a variety of settings.

[I]care for mothers who are pregnant that they have a safe pregnancy. (P1) 
I assess them and part of it is I refer them to the obstetrician for care...this adolescent would have been affected by another disease condition. Um, mostly the sicklers (patients in sickle cell crisis). And she would have ended up in ICU. ... So there isn't much opportunity for teaching. It's mostly giving the postpartum care and doing the best we can do to get them back to wellness. Or to keep them alive. (P2) Or if that individual needs further monitoring in the postnatal period, [...] You should be able to give the care given. We should have flexible laws that should assist us. (P3)

At times, the midwives took on a tone of passion and emotion that became militant. These type of comments illustrated the activist-worker role.

So why couldn't it be the same across the board to wherever we encounter the teenager, the service is the same, nothing changes. So you have equality in the service that's being offered. [...] So if it's one law across the board. [...] stick together and tell them what we want. (P5)

[T] he Midwives Association of Jamaica or the council they need to [consult] stakeholders in the policy forming arena and so they can update policy... [with] evidence-based practice... [and move] towards... The research needs to be done of course. [...] So that's where I think we should start as midwives, research focusing on the adolescents. There could be a controversy there [provision of family planning counseling to a minor] (P4)

\section{The Impact of Culture}

Within the theme of the "impact of culture," the midwives described religion, moral beliefs, and the conservative Jamaican culture that all acted as barriers to the provision of informed consent and participative decision-making. They often acknowledged the WHO policies and International Charter of Rights of the Child, but also acknowledged the a priori laws of the land and status quo in Jamaican society. There was a sense of resignation and pride that "That may be fine for the WHO but this is the way we do things here." or "That doesn't work in Jamaica."

In... our local Jamaican framework, we often times find [they] are also deprived of certain informed choices, because of local cultural, moral and also religious ah beliefs... [T] hese are also barriers to the healthcare provider... (P6)

The culture often caused the perception of adolescents as incapable minors, unable to make decisions for themselves and therefore voiceless. The midwives who considered adolescents capable decision-makers, resilient, and free agents spoke of navigating the dictates of the prevailing Jamaican culture compared to what they verbalized was a more progressive view of adolescents. These midwives had taken on nursing leadership and youth advocacy roles in the community.

When you are speaking to them, they're basically like children, really. And they feel a bithow do I put it-um, in a vulnerable position. They feel vulnerable within that situation, within the health service because one they're teenagers and in their minds they're thinking, "I shouldn't be here." (P5)

One midwife described the internal tension experienced with the recognition that culture and moral beliefs posed a barrier to the midwives allowing the adolescent to have her choice of a desired labor support person. In this case, the desired labor support person was her adult partner and the adolescent found more solace from his labor support than from her own mother.

There was a teenager who was pregnant by her coach at a high school.

...And I was feeling as if I was powerless (to allow her partner in to support her) because I was a senior pupil midwife and these were senior persons to me making this decision.... (P5) 


\section{The Impact of Policy and Place}

Within the theme of "the impact of policy and place," the midwives described the barriers that prevented informed consent of a minor (participative), shared decision-making due to $\mathrm{MoH}$ and hospital policies, and allowance of a desired labor support person due to hospital policy and environmental constraints. They explained that the health policy which categorized adolescent mothers as high-risk patients prevented them from achieving continuity of care with their desired community midwife. Similarly this policy barred them from achieving their desired home birth.

When asked about informed consent, many midwives reported that the current $\mathrm{MoH}$ policy requiring parental consent for adolescents under age 16 was a barrier to assessment and autonomy. However, several midwives were willing to bend the rules to be advocates and to listen to their patients.

...the laws of the land... we should adhere to them. I have seen where we have adolescents in accidents, come into the emergency room and um, decision have to be made. The information isn't forthcoming [from the parents] and so we have had to ask the parents to excuse themselves as they're sometimes becoming hostile and aggressive. We know it's their right because it's a minor... [but]he information given by the adolescent [should] include the adolescent making a decision. So it happens in an informal way and we do know the law of the land...but sometimes you have to adapt to the situation under certain emergent setting. (P2)

[T] here needs to be institutional policies, ... government policies supersedes all of this. [I]nstitutional policies ... [should be] more flexible ... in terms of informed consent ... (P3)

At times, the midwife supported the policies and the severe lack of space in some hospital settings made it more reasonable to abide by the rules. However, for some, the hospital working environment with adolescent mothers held many negative experiences, and evoked strongly held beliefs and feelings.
Yes... we work in the system....in the hospital....And the majority of the teens who go there, we have problems delivering them because of the staffing they don't get support. So we ... end up with problems. But up to last year, we had a home delivery with a teenager and mother and it went so well. (P1)

That's the situation. It brings out the worst in you as a midwife. 'Cause you're placed in that situation and you cannot help um the patient the way you want to. You cannot. Because you have to prioritiz[e]. Yes. So you can't be here to help and provide that support ... [T] he patient leave and say "that nurse this..." because at the time when she's calling you are not able to go. They don't see that. Because they see themselves as being priority. They don't see that this person is not priority at that time, because somebody else [is] over there, but she don't know that. Because she's in the pain and she need you! And you are unable to. So she leave with that feeling..."Nurse this and nurse that." [She leaves with an impression that is] very negative. (P1)

When you said they say "The nurse wicked because you don't come to me right away." And they don't know these people you are looking after at the same time. (P3)

[If I could get a wish, I wish...] we would have to get new buildings. (P1)

Exactly [new buildings]! with more space. So each patient would have a room so they could have their support group present. (P3)

And more workers. (P4)

This part of the text illustrated a perception that both the adolescent mother and the midwife were restricted by the same systemic policies and that the midwife was called upon to be a liberating or emancipatory force providing access to or maintaining choices outside of the policies as depicted in Figure 2. Perron describes the moral distress described by advanced practice nurses (APNs) who observe injustice and health inequity. These APNs are compelled to take on "fearless speech" to advocate for their clients and themselves due to the internal tensions experienced (Perron, Rudge, \& Gagnon, 2014). Examples of frictions and ethical dilemmas include the following: 
But I strongly believe, that the adolescent, if they're rational enough, should be given the right.... [S] ome of them are mature individuals. Even though age [is young]...their thinking capacity might be [mature]...so they should be able to make a rational decision and should be given the opportunity to do so. (very animated). (P3)

[W] e recognize that the law has its place... It would appear as if human rights starts at a particular age, and so, so the teenager, the adolescent is really blocked from making her decision as a human... So then her human right is breached in my opinion. (P3)

One midwife described the double standard which existed where adolescent patients of private clinics were getting around the law and accessed their choice of contraception without parental consent in the private sector, whereas adolescents in the public sector were normally prohibited from informed consent for contraception. This midwife felt more justified to circumvent the policy when such an adolescent presented in the public sector, although she was subjected to greater scrutiny and oversight from the MoH.

The other thing is that my experience at a family planning center. I have administered birth control to teenagers [under age 15] who have come in their uniform from a prominent (expensive, private) high school in Kingston. They turn up and they are actually - it's not a new client (said with emphasis), um. I've actually seen a client who is a part of the clinic has now come in for Depo-provera which I've administered. So the law of the land is there but there is the other side where they go to private practitioners and they are given control. (P2)

[T] here is a need for closer congruence between government policies and policies that are institution-based...having that flexibility and uniformity in offering healthcare and services to our minors. (P6)
You could not just say, "It's the hospital policy we do here." It may be a policy, but if they refuse, they refuse. To me it's like a policy, this is what we do here. So I [tell] them what happens. And most teenagers just nod and go with it. They just go with the flow. (P5)

Navigating these policies produced suffering, confusion, and moral distress when the midwife's strongly held values and beliefs diverted significantly from the policies. Following are the thoughts of a midwife who felt constrained by $\mathrm{MoH}$ and public policies from providing an adolescent with her choice of contraception because her mother's choice had to prevail over the adolescent's choice. In this case, informed consent of a minor could not be fulfilled due to the current emancipated minor law which makes age 16 the age of accountability.

Sometimes you have a minor that comes to you, we would have deemed, this would have been ideally one of the most appropriate methods for her. However, I get a sense of disappointment as though the system has failed this young woman. Because, first of all, internationally there are other options that she could have. If she were elsewhere, but already she is faced with limited choices in the healthcare system. (P6)

Along with choice of contraception for minors, choosing home as a place of birth was restricted for all adolescents up until the age of 18 at which time they were considered adults and no longer high-risk patients. Also, urban nurse-midwives practicing in hospital-based clinics and attending hospital births verbalized more restrictions and adherence to institutional policies than the rural community midwives who practiced in community clinics and attended homebirths. Consequently, the place where midwifery was practiced was a determinant of how many barriers were faced in respectful care provision.

At times, the general public policies also prevented the types of family planning education that the midwives believed was fair and necessary in the community. The midwives concluded that these policies were barriers to the adolescent girls' decision-making power. 
They (nurse educators) go to the school. There's a start of that in Jamaica. There was controversy as to whether we should introduce condoms in school...so teenage boys. Since the boys are kept in school but the girls once they're pregnant they're asked to leave school. (P4)

[Following policy and preventing her from starting contraception] ... is going to put her back into a vulnerable situation without providing her at the least the basic options. So that is what [happens at] times. And for me it's a very disappointing, disheartening feeling. (Pause). (P6)

The midwives identified the following emotions that were tied to larger themes that underlay and drove action. Following are excerpts of their verbalizations: "I felt ....powerless..."; "I felt disappointed..."; “it was... disappointing"; "it was ...disheartening..." Many of the midwives verbalized beliefs and values that were divergent from those of the health or public policies and prevailing institutional rules by which they were constrained at work.

They also emoted strongly held feelings and beliefs that were either congruent with the mothers or divergent from the mothers.

\section{DISCUSSION}

To summarize, the poststructural feminist version of Denzin's interpretive interactionism theory can easily be applied to the findings of this pilot study which is depicted in Figure 1. This graphic is the researcher's interpretation of symbolic interactions with significant others in the Jamaican midwife's life as these descriptions emerged in the interviews. The two concentric circles represent a circle of influence on the midwife. The I/ME represents the significant experiences when the midwife and key persons interact with each other. Jamaican Society is comprised of social or public policy, health policy, culture, norms, economy, and rules of engagement during work and care delivery.

The participants explained that the influence of values and roles were easily overcome by the power of policy and place in ways that restricted the ability of midwives to provide respectful care by the WHO definition. This tug-of-war process resulted in pivotal, emotional work experiences for the midwives. For some (both rural and community midwives), these emotions fed into their self-perceptions of activists and change agents at work or in the community. These feelings fueled their motivations to circumvent policies and to take on a fearless voice on behalf of their clients (Perron et al., 2014). It was difficult but necessary for the researcher to simply listen and to bracket feelings of empathy for the midwives who described stories of working in challenging low resource settings. The participants echoed issues that have been raised in the growing literature about sexual health and reproductive rights for adolescents. As Denzin described, both the researcher and the participants can partner when both sense an obligation to improve quality care, as well as health and social outcomes. The participants' strongly held beliefs and values did influence both their experience while providing care and their behaviors with regards to respectful care. Furthermore, the researcher had an obligation to contribute in a meaningful way to quality improvement by utilizing the research findings.

There was tremendous congruence among the midwives of the group in their beliefs and it may be possible that saturation had been reached for these five questions. Most of the rural midwives reported ways in which they were flexible or would modify rules to continue caring for adolescents even though adolescents were deemed high-risk and outside of their scope in the community practice. In contrast, only a few of the urban nurse-midwives reported these types of sentiments or advocacy activities. Consequently, place of work appeared to be associated with advocacy behaviors.

The area in which there was divergence of opinions was the general acceptance of the controversial hospital policies by some of the urban nurse-midwives. Two hospital-based midwives who had private interviews believed it was simply their job to obey the rules and they had no internal dismay about the lack of provision of informed consent, sharing decision-making, or denying access to a labor support companion. The sense of duty to adhere to policy was not restricted to the midwives who were working in an obstetrical nurse role. And all midwives in the study were either currently attending adolescent births or had attended in the past. It is possible that their peers in focus groups might secretly have felt the same way as the two participants in the private interviews. However, they may have been subject to the desire to maintain group cohesion or a reluctance to relay their true feelings in the group. There was one nurse-midwife who, in particular, openly reflected on her previous reluctance to consider adolescent mothers 
as having the right to self-determination but later began to notice how much her past religious beliefs had influenced her views. She shared an interesting experience of revelation, self-reflection, and examination of the underpinnings and values that fuel treatment of adolescents. She also shared that her beliefs were shaped by her experience of loss and grief after caring for high-risk postpartum mothers on ventilators who had succumbed to the complications of sickle-cell disease.

There was one surprising finding in this study. Although the informed consent could not promise any benefit from participation in the study, all the participants reported feelings of gratitude, satisfaction, and some degree of validation after their participation in the focus groups. Others reported that the focus groups provided a welcomed opportunity for self-reflection and support for their community advocacy work. It is possible that the feminist approach of minimizing power differentials and the cultural safety achieved in focus group discussions provided a source of empowerment in contrast to the disempowering environment often described in the facilities due to restrictive policies.

Limitations of the study include the small sample size. The timing and geographic distances required to reach the larger number of busy midwives across the mountainous terrain of Jamaica limited the number of rural midwives sampled. Many of the midwives reported that they did not receive ample communication about the conference and there were likely dozens more that were invited to come but could not attend on such short notice. In future, a snowballing sampling technique and use of Skype or telephone interviews could increase recruitment and the study to reach saturation for each theme (Creswell, 2012, p. 135) structured interviews (Deakin \& Wakefield, 2013; Sullivan, 2013). Most of the midwives reported that they had Internet access in their homes or on their smartphones, although they lacked Internet access at their clinics. The challenge of traveling for the focus groups could be alleviated with Skype focus groups hosted from the researcher's private office in which the digital recorder captures the voices on the speakers and the researcher may see the facial expressions of the participants and record them for analysis.

Other limitations of the study were the wording of the question about privacy and the concept of choice being interpreted differently in the Jamaican vernacular. Vetting the interview questions with key informants or currently practicing Jamaican midwives prior to data collection would have been helpful. This would have promoted improved comprehension for the participants. Researchers have explored the challenges of working across different linguistic and cultural meanings with respect to health literacy and care delivery (Lori, Munro, \& Chuey, 2016) as well as health-related research when both the researcher and participants speak the same language (Al-Amer et al., 2016). Some of the emerging reproductive rights issues raised by this study have yet to be described or explored by practicing nurses and midwives in the Global South. Both the researcher and the participants could identify that the adolescent was vulnerable; however, the Jamaican culture as well as the social policies continue to treat adolescents as children or minors who should be protected by making decisions for them. When the definition of choice was described as provision of a choice that the adolescent mother would make for herself, it was perceived instead as the decision that the midwife was obligated to make for the adolescent since she was incapable. Choice has very different conceptualizations when comparing Jamaican to North American ways of knowing. Without any context to use, the concept of choice is difficult to capture when working across such a cultural difference. Consequently, there were unexpected outcomes to the project.

Very rarely did the midwife ever affirm that she would normally invite the adolescent mother to share her desires or wishes. None of the other examples of shared decision-making that are common in North American hospital environments seemed to be comprehended by the participants (e.g., alternative laboring or delivery position, declining blood tests, requesting a labor companion, various choices of family planning). When the scenario of an adolescent arriving with a request for a particular type of contraceptive method was presented, often this scenario was not interpreted as the adolescent making a choice. Instead it was perceived as the adolescent requesting education, rather than information. With many participants, the adolescent was not seen as an individual with agency or self-efficacy, but a vulnerable person unable to make choices, who required education and decision-making by the adult midwife. In addition, the Jamaican healthcare system and culture are paternalistic by nature. Because there was so much variance in the way the participants comprehended it, question number three should be reconstructed for future study replication.

The privacy question did not evoke much discussion and should probably be replaced with another more relevant ethical right in the WHO definition. The midwives implied that this tenet was emphasized strongly in their education and patient issues were only discussed in professional environments for the purposes of case 
review, inter- or intra-professional consultations or care planning.

Despite the researcher's familiarity with Jamaican culture and vernacular, as well as the use of iterative questioning, definitions, and elaborations when requested, another limitations in the methodology was the absence of triangulation which is commonly used to maximize credibility in the detailed methodology used by grounded theorists. Although time-consuming, it might be necessary to change the methodology from a generic qualitative study to a more complex study using a seven-step method of Colaizzi, which would allow the researcher to revisit participants to validate the meanings of their words, particularly in light of the various meanings of new concepts such as shared decision-making. Grounded theory methods would also allow development of theoretical constructs for how the concepts are connected and related or help to determine what facilitates respectful care (McCreaddie \& Payne, 2010).

Replicability of the project in other settings would also strengthen credibility and transferability of this study (Shenton, 2004). There is an opportunity for the project to be replicated to achieve saturation by interviewing midwives throughout the Caribbean and Central America as part of a larger youth and child rights project. It could inform policy that mitigates or removes current barriers to respectful care, as recommended by Bohren and colleagues (Bohren et al., 2015).

\section{CONCLUSIONS}

In many cases, what the teens experienced as infringements on choice, decision-making, dignity, and selfesteem were unintentional and could illustrate cultural stigma, lack of knowledge, or skill in the provision of respectful care vis-a-vis respect for sexual and reproductive health rights as defined by WHO (Abuya et al., 20152012, p. 3/13; Bowser \& Hill, 2010, p. 11). This study provided a peek into the perspectives and opinions concerning these behaviors by Jamaican midwives who care for adolescent mothers adding to the sparse literature describing RMC of mothers in the Caribbean. Midwives have identified that health policy and cultural beliefs have been significant barriers to providing respectful care that involves choice, informed consent, and a desired labor support person, particularly in the hospital setting. They see their roles as advocates, educators, expert caregivers, and activists who have found a political voice to espouse the rights of their adolescent clients. Study findings cannot be generalized; however the study should be replicated throughout the Caribbean. The findings may be used to inform a national midwifery survey, educational modules, quality improvement, health systems change, and youth empowerment strategies. In addition, the findings may inform future policy analysis and recommendations for changes that support adolescent reproductive and sexual health rights in Jamaica.

This pilot was seminal and there remains a paucity of research on the perspectives of adolescent mothers about respectful care. In future, the authors recommend additional exploration, discourse analysis, and concept analysis that develop the context of the work life and work environment of the midwife. In addition, research exploring the lived experiences of adolescent mothers and the prevalence of disrespectful care and abuse should be measured. This work would perhaps inform future national surveys to determine which variables correlate with disrespectful behaviors in maternity care. A feminist lens was valuable for setting up a culturally safe space for focus group discussion and for analysis of the effect of institutional policies on midwife workers. In future, it would be relevant to analyze their workspaces using institutional ethnography (Smith, 2009) to reveal how gendered spaces such as maternity units create restricted agency which selectively and systemically discriminates against vulnerable groups such as female adolescent clients and primarily female midwife workers.

\section{REFERENCES}

Abuya, T., Warren, C. E., Miller, N., Njuki, R., Ndwiga, C., Maranga, A., . . . Bellows, B. (2015). Exploring the prevalence of disrespect and abuse during childbirth in Kenya. Plos One, 10(4), e0123606-e0123606. http://dx. doi.org/10.1371/journal.pone.0123606

Aguilar, M. (2015). Region must invest in reducing adolescent pregnancies - UNFPA official The Jamaican Observer. Retrieved from http://www.jamaicaobserver.com/news/ Region-must-invest-in-reducing-adolescent-pregnancies---UNFPA-official

Al-Amer, R., Ramjan, L., Glew, P., Darwish, M., \& Salamonson, Y. (2016). Language translation challenges with Arabic speakers participating in qualitative research studies. International Journal of Nursing Studies, 54, 150-157. 
Alliance, W. R. (2015). Respectful maternity care: A Nigeriafocused health worker's training guide. Washington, DC: Futures Group, Health Policy Project.

Atuyambe, L., Mirembe, F., Annika, J., Kirumira, E. K., \& Faxelid, E. (2009). Seeking safety and empathy: Adolescent health seeking behavior during pregnancy and early motherhood in central uganda. Journal of Adolescence, 32(4), 781-796. http://dx.doi.org/10.1016/j.adolescence.2008.10.012

Bhutta, Z. A., Soofi, S., Cousens, S., Mohammad, S., Memon, Z. A., Ali, I., \& Martines, J. (2011). Improvement of perinatal and newborn care in rural Pakistan through community-based strategies: A cluster-randomised effectiveness trial. The Lancet. Retrieved from http:// search.proquest.com.frontier.idm.oclc.org/docview/ 856796142 ? accountid $=10993$

Bohren, M. A., Vogel, J. P., Hunter, E. C., Lutsiv, O., Makh, S. K., Souza, J. P., . . Gülmezoglu, A. M, Javadi, D. (2015). The Mistreatment of Women during Childbirth in Health Facilities Globally: A Mixed-Methods Systematic Review. PLOS Medicine, 12(6), e1001847. http://dx. doi.org/10.1371/journal.pmed.1001847

Bonenberger, M., Aikins, M., Akweongo, P., \& Wyss, K. (2014). The effects of health worker motivation and job satisfaction on turnover intention in Ghana: A cross-sectional study. Human Resources for Health, 12, 43-43. http://dx.doi.org/10.1186/1478-4491-12-43

Bowser, D., \& Hill, K. (2010). Exploring evidence for disrespect and abuse in facility-based childbirth. Boston, MA: USAID-TRAction Project, Harvard School of Public Health.

Braun, V., \& Clarke, V. (2006). Using thematic analysis in psychology. Qualitative research in psychology, 3(2), 77-101. http://dx.doi.org/10.1191/ 1478088706qp063oa

Creswell, J. W. (2012). Qualitative inquiry and research design: Choosing among five approaches (4th ed). Thousand Oaks, CA: Sage.

Dao, B. (2012). Guidelines for in-service training in basic and comprehensive emergencyobstetric and newborn care. Baltimore: Jhpiego. Retrieved from http://whiteribbonalliance.org/wp-content/uploads/2013/09/RMCGuide_FINAL.pdf

Deakin, H., \& Wakefield, K. (2013). Skype interviewing: Reflections of two $\mathrm{PhD}$ researchers. Qualitative Research, 1468794113488126.

Denzin, N. K. (2001). Interpretive interactionism (2nd ed., Vol. 16). Thousand Oaks, CA: Sage Publications Inc.

Duggan, R., \& Adejumo, O. (2012). Adolescent clients' perceptions of maternity care in KwaZulu-natal, South Africa. Women and Birth: Journal of the Australian Col- lege of Midwives, 25(4), e62-e67. http://dx.doi.org/10. 1016/j.wombi.2011.10.004

Gee, R. E., \& Corry, M. P. (2012). Patient engagement and shared decision making in maternity care. Obstetrics and Gynecology, 120(5), 995-997. http://dx.doi.org/ http://10.1097/AOG.0b013e31827046ac

Hutchinson, M. K., Kahwa, E., Waldron, N., Hepburn Brown, C., Hamilton, P. I., Hewitt, H. H., . . . Sweet Jemmott, L, Brown, C. H, Jemmott, L. S. (2012). Jamaican mothers' influences of adolescent girls' sexual beliefs and behaviors. Journal of Nursing Scholarship, 44(1), 27-35. http://dx.doi.org/10.1111/j.1547-5069.2011. 01431.x

James, S., Rall, N., \& Strümpher, J. (2012). Perceptions of pregnant teenagers with regard to the antenatal care clinic environment. Curationis, 35(1), 1-8. http://dx. doi.org/10.4102/curationis.v35i1.43

Jefford, E., \& Sundin, D. (2013). Post-structural feminist interpretive interactionism. Nurse Researcher, 21(1), 14-22. http://dx.doi.org/10.7748/nr2013.09.21.1.14.e303

Knight, H. E., Self, A., \& Kennedy, S. H. (2013). Why are women dying when they reach hospital on time? A systematic review of the 'third delay'. PLoS One, 8(5), e63846. http://dx.doi.org/10.1371/journal.pone. 0063846

Lori, J. R., Munro, M. L., \& Chuey, M. R. (2016). Use of a facilitated discussion model for antenatal care to improve communication. International Journal of Nursing Studies, 54, 84-94. http://dx.doi.org/10.1016/j.ijnurstu. 2015.03.018

McCreaddie, M., Payne, S (2010). Evolving grounded theory methodology: Towards a discursive approach. International Journal of Nursing Studies, 47(6), 781-793. http:// dx.doi.org/10.1016/j.ijnurstu.2009.11.006

Miller, S., Cordero, M., Coleman, A. L., Figueroa, J., Brito-Anderson, S., Dabagh, R., . . Nunez, M. (2003). Quality of care in institutionalized deliveries: the paradox of the Dominican Republic. International Journal of Gynecology \& Obstetrics, 82(1), 89-103. http://dx.doi.org/10. 1016/S0020-7292(03)00148-6

Miller, S., Abalos, E., Chamillard, M., Ciapponi, A., Colaci, D., Comandé, D., ... Althabe, F, Manuelli, V. (2016). Beyond too little, too late and too much, too soon: a pathway towards evidence-based, respectful maternity care worldwide. The Lancet, 388(10056), 2176-2192. http://dx.doi.org/10.1016/S0140-6736(16)31472-6

Morgan, D. L. (1998). The focus group guidebook. Thousand Oaks, CA: Sage.

Murray, C. J., Vos, T., Lozano, R., Naghavi, M., Flaxman, A. D., Michaud, C., . . Memish, Z. A, Grant, B. (2012). Disability-adjusted life years (DALYs) for 291 diseases 
and injuries in 21 regions, 1990-2010: a systematic analysis for the Global Burden of Disease Study 2010. The Lancet, 380(9859), 2197-2223. http://dx.doi.org/ 10.1016/S0140-6736(12)61689-4

Oyerinde, K., Harding, Y., Amara, P., Garbrah-Aidoo, N., Kanu, R., Oulare, M., . . Daoh, K. (2013). A qualitative evaluation of the choice of traditional birth attendants for maternity care in 2008 Sierra Leone: Implications for universal skilled attendance at delivery. Maternal and Child Health Journal, 17(5), 862-868. http://dx.doi. org/10.1007/s10995-012-1061-4

Perron, A., Rudge, T., \& Gagnon, M. (2014). Towards an "ethics of discomfort”. In P. N. Kagan, M. C. Smith, \& P. L. Chinn (Eds.), Philosophies and practices of emancipatory nursing: Social justice as praxis. London, NY: Routledge.

Prost, A., Colbourn, T., Seward, N., Azad, K., Coomarasamy, A., Copas, A., . . Costello, A. (2013). Women's groups practising participatory learning and action to improve maternal and newborn health in low-resource settings: A systematic review and meta-analysis. The Lancet, 381(9879), 1736-1746. http://dx.doi.org/10.1016/ S0140-6736(13)60685-6

Shenton, A. K. (2004). Strategies for ensuring trustworthiness in qualitative research projects. Education for Information, 22(2), 63-75.

Shiferaw, S., Spigt, M., Godefrooij, M., Melkamu, Y., \& Tekie, M. (2013). Why do women prefer home births in Ethiopia? BMC Pregnancy and Childbirth, 13, 5-5. http://dx.doi.org/10.1186/1471-2393-13-5

Singh, P. K., Rai, R. K., Alagarajan, M., \& Singh, L. (2012). Determinants of maternity care services utilization among married adolescents in rural India. PLoS One, 7(2), e31666n/a. http://dx.doi.org/10.1371/journal. pone.0031666

Small, R., Yelland, J., Lumley, J., Brown, S., \& Liamputtong, P. (2002). Immigrant women's views about care during labor and birth: An Australian study of Vietnamese, Turkish, and Filipino women. Birth: Issues in Perinatal Care, 29(4), 266-277. Retrieved from http://dx.doi.org/ 10.1046/j.1523-536X.2002.00201.x

Sullivan, J. R., \& Sullivan, J. R. (2013). Skype: An appropriate method of data collection for qualitative interviews? The Hillop Review, 6(1), 10.

Sundin, D., \& Fahy, K. (2008). Critical, post-structural, interpretive interactionism: An update on Denzin's methodology. Nurse Researcher, 16(1), 7-23. http://dx. doi.org/10.7748/nr2008.10.16.1.7.c6750
Thompson, J. E., Land, S., Camacho-Hubner, A. V., \& Fullerton, J. T. (2015). Assessment of provider competence and quality of maternal/newborn care in selected Latin American and Caribbean countries. Revista panamericana de salud publica $=$ Pan American journal of public health, 37(4-5), 343-350.

Tuckett, A. G. (2005). Applying thematic analysis theory to practice: a researcher's experience. Contemporary Nurse, 19(1-2), 75-87.

United Nations Population Fund. (2013). Jamaica offers a model for preventing adolescent pregnancies while supporting young mothers. Retrieved from http:// unfpa.org/public/home/news/pid/14825\#sthash.uQ9agiHK.dpuf (Accessed cited 2014 2/2/2014).

Vieira, C. L., Coeli, C. M., Pinheiro, R. S., Brandão, E. R., Camargo, K. R., Aguiar, F. P (2012). Modifying effect of prenatal care on the association between young maternal age and adverse birth outcomes. Journal of Pediatric and Adolescent Gynecology, 25(3), 185-189. http://dx. doi.org/10.1016/j.jpag.2011.12.070

Wilson-Mitchell, K. (2015). Midwives perceptions of barriers to quality maternity care forJamaican adolescents. Retrieved from https://frontier.contentdm.oclc.org/ digital/collection/dc/id/609/rec/1Windau-Melmer

Wilson-Mitchell, K., Bennett, J., Stennett, R (2014). Psychological health and life experiences of pregnant adolescent mothers in Jamaica. International Journal of Environmental Research and Public Health, 11(5), 4729-4744. http://dx.doi.org/10.3390/ ijerph110504729

Wilson-Mitchell, K., \& Stevens, R. (2014). An innovative teen-centered antenatal care model compared to standard antenatal care in Jamaica. Global Journal of Human Social Science, 14(3), 53-61.

World Health Organization. (2015a). Selected policies on adolescent friendly health services in low and middle income countries.

World Health Organization. (2015b). Sexual health, human rights and the law. Retrieved from http://apps.who.int/ iris/bitstream/10665/175556/1/9789241564984_eng. pdf?ua $=1$

Wilson-Mitchell, K. (2016). Midwives' perceptoins of barriers to quality maternity care for Jamaican adolescents. Doctoral Captstone Project. Retrieved from http://cdm16161.contentdm.oclc.org/cdm/search/ collection/dc/searchterm/Wilson-Mitchell/order/ nosort 
Correspondence regarding this article should be directed to Karline Wilson-Mitchell, DNP, MSN, CNM, Ryerson University, Toronto, Ontario, Canada. E-mail: k.wilsonmitchell@ ryerson.ca

Karline Wilson-Mitchell, DNP, MSN, CNM, Associate Professor, Ryerson University, Toronto, Ontario, Canada.
Amy Marowitz, DNP, CNM, Associate Professor, Frontier Nursing University, Hyden, KY.

Jody R. Lori, PhD, CNM, FACNM, FAAN, Associate Professor, University of Michigan, Associate Dean for Global Affairs, Ann Arbor, MI. Former Chair Global Health Division, ACNM Director, PAHO/WHO Collaborating Center. 